\title{
On-line scavenging of trace analytes in complex matrices for fast analysis by carbon dioxide bubbling extraction coupled with gas chromatography-mass spectrometry
}

Yuanji Gao ${ }^{\mathrm{a}, *}$, Bing Xia ${ }^{\mathrm{b}}$, Yunan Qin ${ }^{\mathrm{a}}$, Ke Huang ${ }^{\mathrm{a}}$, Congling Yang ${ }^{\mathrm{a}}$, Qing Yang ${ }^{\mathrm{a}}$ and Lijuan Zhao ${ }^{\mathrm{a}}$

${ }^{a}$ College of Chemistry and Materials Science, Sichuan Normal University, Chengdu 610068, P.R. China

${ }^{\mathrm{b}}$ Chengdu Institute of Biology, Chinese Academy of Sciences, No. 93 South Keyuan Road, Gaoxin Distinct, Chengdu 610041, P. R. China

*Corresponding Author. Tel: +86 15002850841, Fax: +86 02884760804 . E-mail address: yuanjijoy@163.com 


\section{Table of Contents}

S-1. Optimization of $\mathrm{CO}_{2}$ bubbling extraction conditions............................ S-3

S-2. TOC analysis with CDBES-GC-MS................................................. S-4

S-3. Volatiles in the hops analyzed with CDBES-GC-MS............................ S-5

S-4. Volatiles in the urine sample analyzed with CDBES-GC-MS.................... S-6

Table S1. The retention time $\left(t_{R}\right)$, quantification, and confirmation ions of the 8

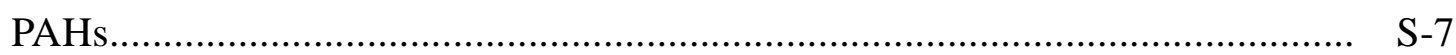

Table S2. The operating parameters of ICP-MS ............................................ S-7

Table S3. Quantitative results of the 8 PAHs with CDBES-GC-MS/MS........... S-8

Table S4. Identification of PAHs in tap water, tap water spiked with 10 ng of 8 PAHs, and a soil sample with CDBES-GC-MS................................................. S-8

Table S5. The comparison of main volatiles (>1.0\%) with different sample preparation techniques.

Table S6. Volatiles in lemonade bubble iced coffee analyzed with CDBES-GC-MS showing the retention time, name, formula, CAS, and percentage of matching score of each compound according to the NIST11 database.

S-10

Table S7. Volatiles found in the urine analyzed with CDBES-GC-MS showing the retention time, name, formula, CAS, and percentage of matching score of each compound according to the NIST11 database.

Figure S1. The overall SNR trend of bubbling extraction with different carrier gases: (a) $\mathrm{CO}_{2}$, (b) $\mathrm{CH}_{4}$, (c) $\mathrm{N}_{2}$, (d) $\mathrm{He}$, and (e) Ar.

Figure S2. Influence of the parameters on the $\mathrm{CO}_{2}$ bubbling extraction efficiency using $50 \mu \mathrm{g} / \mathrm{mL}$ standard PAHs in a spiked water solution. (a) ionic strength effects: extraction temperature of $50{ }^{\circ} \mathrm{C}$, bubbling time of $15 \mathrm{~min}$ and $\mathrm{CO}_{2}$ flow rate of $100 \mathrm{~mL} / \mathrm{min}(n=$ 3); (b) extraction temperature effects: $4.0 \mathrm{~g}$ of $\mathrm{NaCl}$, bubbling time of $15 \mathrm{~min}$ and $\mathrm{CO}_{2}$ flow rate of $100 \mathrm{~mL} / \mathrm{min}(n=3)$; (c) effects of $\mathrm{CO}_{2}$ flow rate: extraction temperature of $50{ }^{\circ} \mathrm{C}, 4.0 \mathrm{~g}$ of $\mathrm{NaCl}$ and bubbling time of $15 \mathrm{~min}(n=3)$; and (d) effects of bubbling time: $\mathrm{CO}_{2}$ flow rate of $100 \mathrm{~mL} / \mathrm{min}$, extraction temperature of $50^{\circ} \mathrm{C}$ and $4.0 \mathrm{~g}$ of $\mathrm{NaCl}$ $(n=3)$. S-14

References. S-14 


\section{S-1. Optimization of $\mathrm{CO}_{2}$ bubbling extraction conditions}

The concentrations of the analytical standards were in the range of $10.0-100 \mu \mathrm{g} / \mathrm{L}$, and the concentration of internal standards (ISs) was $20.0 \mu \mathrm{g} / \mathrm{L}$. The coefficients of determination $\left(R^{2}\right)$ were in the range of $0.991-0.995$ (Table S3). Here, CDBES was established as an ideal technique for the measurement of target compounds, including volatile/semivolatile analytes, metal ions, inorganic salts, and metal-organic compounds.

Here, we investigated the effect of sodium chloride $(\mathrm{NaCl})$ at varied concentrations on the extraction efficiency of the test PAHs. The influence of ionic strength on the extraction performance was characterized at $0,2,4,6,8$, and $10 \mathrm{~g} \mathrm{NaCl}$ per vial using $50 \mathrm{ng} / \mathrm{mL}$ standard analyte in aqueous solution (Figure S2a). The extracted masses of analytes increased with the ionic strength because of the decreased solubility of the 8 PAHs. The adsorption of PAHs reached equilibrium with the $\mathrm{NaCl}$ concentration at 4.0 g per vial, which was selected as the optimal value for ionic strength.

As the bubbling time increases up to $15 \mathrm{~min}$, a plateau in peak area is reached (Figure S2d). Thus, 15 minutes represents the time required for more and more target analytes to be transferred from aqueous media to the trap and for the system to reach equilibrium, and this time is considered the optimized bubbling time. However, within a short time (2 min) of $\mathrm{CO}_{2}$ bubbling extraction, the peak area values of some analytes suddenly increased, perhaps because of the boiling bubble effect of the carrier gas. To achieve the best compromised conditions for the $8 \mathrm{PAH}$ analytes, 2.0 min was selected as the most optimal bubbling time. 
The $\mathrm{CO}_{2}$ bubbling extraction temperature can also be an important factor in organic fraction domination. ${ }^{1}$ Sample temperature is another important parameter for optimizing the extraction efficiency. Higher sample temperatures enhance the extraction efficiency in reduced time because of accelerated molecular motion. However, an excessively high sample temperature may cause bigger drops of aerosol to slide down the internal wall of the U-type chamber due to its own gravity. The influence of sample temperature on the purge efficiency was characterized at 40, 50, 60, 70, 80, and $90^{\circ} \mathrm{C}$ using a $50 \mathrm{ng} / \mathrm{mL}$ standard water solution (Figure S2b). The response of the 8 PAHs decreased as the sample temperature increased because of the increased volatility of the water at higher temperatures. The adsorption of the PAHs reached equilibrium at a sample temperature of $50{ }^{\circ} \mathrm{C}$. Therefore, $50{ }^{\circ} \mathrm{C}$ was selected as the optimal sample temperature.

Another important parameter is the $\mathrm{CO}_{2}$ flow rate. If the gas flow rate is too low, target components may not be extracted into the trap. As shown in Figure S2c, extraction efficiency gradually increased with the increase of flow rate. Limited by the pressure of dry ice gas, $100 \mathrm{~mL} / \mathrm{min}$ was selected as the optimal flow rate of $\mathrm{CO}_{2}$.

During each GC-MS measurement cycle, the sampling needle, loop, and U-type chamber were cleaned with hot water $\left(90^{\circ} \mathrm{C}\right)$. The trap was also heated at $250{ }^{\circ} \mathrm{C}$ for $8.0 \mathrm{~min}$. These procedures were sufficient to clean the purge system for subsequent measurements. The dry purge step was set to 2.0 min to remove water trapped on the sorbent.

\section{S-2. TOC analysis with CDBES-GC-MS}


TOC in the water is believed to originate primarily from natural, humic substances from plant and plant residues. These substances include humin, humic acid and fulvic acid. No requirement is more frustrating to many operators of surface water treatment plants than the total organic carbon (TOC) percentage removal requirement. It is necessary to remove TOC from aquaculture wastewater using $\mathrm{CO}_{2}$ extraction technology. To monitor the organics in water with an absorbance system, potassium acid phthalate (KHP) solution at five different concentrations (2.0, 5.0, 10, 20, and 40 mg/L) was prepared as a standard sample for TOC testing by dissolving KHP powder $\left(\mathrm{C}_{8} \mathrm{H}_{5} \mathrm{KO}_{4}\right)$ in distilled water. After $\mathrm{CO}_{2}$ purging for 20 min, TOC detected by KHP dropped by $31.3 \%$.

\section{S-3. Volatiles in the hops analyzed with CDBES-GC-MS}

The volatiles in hops were analyzed by the headspace (HS), headspace solid phase microextraction (HS-SPME) ${ }^{2}$ and dynamic headspace (DHS) ${ }^{3}$ methods under the same conditions $(0.1 \mathrm{~g} / 10 \mathrm{~mL}$ aqueous solution), and the comparison of main volatiles $(>1.0 \%)$ is shown in Table S5. In HS-injection mode, a CTC autosampler (Pal RTC, CTC analytics, Switzerland) was used with a Headspace static tool in splitless mode. The incubation parameters were set as follows: 10 min incubation time in agitator mode at a temperature of $50{ }^{\circ} \mathrm{C}$ and speed of $500 \mathrm{rpm}$. The injection volume was $500 \mu \mathrm{L}$. The

fibers used for HS-SPME tests were polydimethylsiloxane/divinylbenzene (PDMS/DVB, $65 \mu \mathrm{m}$ ) purchased from Supelco (Sigma-Aldrich, Bellefonte, PA, USA). A CombiPAL Autosampler (CTC Analytics, Zwingen, Switzerland) was used with the following conditions: incubation temp, $50^{\circ} \mathrm{C}$; injection volume, $22 \mu \mathrm{L}$; extraction time, 
10 min; agitator speed, 500 rpm; desorption time, 3 min. For DHS, the analytes were purged from aqueous solution at $55{ }^{\circ} \mathrm{C}$ for $10 \mathrm{~min}$ in nitrogen at $40 \mathrm{~mL} / \mathrm{min}$ and were absorbed on the trap. The dry purge step was set to 2 min to remove water trapped on the sorbent. The trap temperature was subsequently rapidly increased to $245^{\circ} \mathrm{C}$ in the absence of carrier gas flow. Finally, the trapped components were desorbed at $250{ }^{\circ} \mathrm{C}$ for 3 min and transferred directly to the GC-MS for analysis.

\section{S-4. Volatiles in the urine sample analyzed with CDBES-GC-MS}

Pretreatment of urine samples is usually required before analysis. However, large quantities of inorganic constituents are detrimental to chromatographic columns and MS instrumentation. Lyophilization and chemical extraction also suffer from chemical losses. A popular sample pretreatment approach in urine analysis is the simple dilution (e.g., 100-1,000-fold) of urine samples in water or organic solvent. ${ }^{4}$ However, sample dilution also results in the decreased detectability of chemicals present in urine at trace concentrations. Urine can be analyzed by $\mathrm{CO}_{2}$ bubbling extraction, as shown in Table S6. Thirty-three volatile metabolites were found in the urine sample. The effect of volatile preconcentration is important for increasing the chemical sensitivity of urine analysis and is also useful for monitoring toxic and prohibited pharmaceutical molecules present in urine at trace levels. 
Table S1. The retention time $\left(\mathrm{t}_{\mathrm{R}}\right)$, quantification, and confirmation ions of the 8 PAHs.

\begin{tabular}{|c|c|c|c|c|c|c|}
\hline Analytes & Struture & Abbreviation & $t_{R}(\min )$ & $\begin{array}{c}\text { Quantitative } \\
\text { ion }(\mathrm{m} / \mathrm{z})\end{array}$ & $\begin{array}{l}\text { Qualitative ion } \\
\qquad(\mathrm{m} / \mathrm{z})\end{array}$ & IS \\
\hline Naphthalene & & NP & 5.769 & 128 & 108,154 & Naphthalene- $d_{8}$ \\
\hline Acenaphthylene & & ANP & 7.810 & 152 & 151,153 & $\begin{array}{c}\text { Acenaphthylene- } \\
\qquad d_{10}\end{array}$ \\
\hline Acenaphthene & & ANE & 8.107 & 154 & 153,152 & $\begin{array}{c}\text { Acenaphthylene- } \\
\qquad d_{10}\end{array}$ \\
\hline Fluorene & & FLR & 9.101 & 166 & 165,167 & $\begin{array}{c}\text { Acenaphthylene- } \\
\qquad d_{10}\end{array}$ \\
\hline Phenanthrene & & PHE & 11.962 & 178 & 179, 176 & Fluorene- $d_{10}$ \\
\hline Fluoranthene & & FLT & 16.314 & 202 & 200, 203 & Fluorene- $d_{10}$ \\
\hline Pyrene & & PY & 16.936 & 202 & 200, 203 & Fluorene- $d_{10}$ \\
\hline Chrysene & & CHR & 20.468 & 228 & 226, 229 & Fluorene- $d_{10}$ \\
\hline
\end{tabular}

Table S2. The operating parameters of ICP-MS.

\begin{tabular}{ll}
\hline Parameters & Value \\
\hline Radio frequency power & $1550 \mathrm{~W}$ \\
Coolant argon gas flow rate & $1.0 \mathrm{~mL} / \mathrm{min}$ \\
Carrier (nebulizer) gas flow rate & $15 \mathrm{~mL} / \mathrm{min}$ \\
Auxiliary argon gas flow rate & $1.0 \mathrm{~mL} / \mathrm{min}$ \\
Scanning mode & Peak area \\
Dwelling time & $40 \mathrm{~s}$ \\
& $107 \mathrm{Ag},{ }^{75} \mathrm{As},{ }^{137} \mathrm{Ba},{ }^{9} \mathrm{Be},{ }^{43} \mathrm{Ca},{ }^{44} \mathrm{Ca},{ }^{112} \mathrm{Cd}$, \\
Isotope monitored & ${ }^{59} \mathrm{Co},{ }^{52} \mathrm{Cr},{ }^{133} \mathrm{Cs},{ }^{71} \mathrm{Ga},{ }^{39} \mathrm{~K},{ }^{7} \mathrm{Li},{ }^{24} \mathrm{Mg},{ }^{55} \mathrm{Mn}$, \\
& ${ }^{23} \mathrm{Na},{ }^{207} \mathrm{~Pb},{ }^{206} \mathrm{~Pb},{ }^{208} \mathrm{~Pb}{ }^{85} \mathrm{Rb},{ }^{78} \mathrm{Se},{ }^{88} \mathrm{Sr}$, \\
\hline & ${ }^{205} \mathrm{Tl},{ }^{238} \mathrm{U}$ and ${ }^{51} \mathrm{~V}$ \\
\hline
\end{tabular}


Table S3. Quantitative results of the 8 PAHs with CDBES-GC-MS/MS.

\begin{tabular}{cccccc}
\hline Analytes & Linearity range $(\mu \mathrm{g} / \mathrm{L})$ & $R^{2}$ & $\mathrm{RSD}(n=6)$ & $\mathrm{LOD}(\mu \mathrm{g} / \mathrm{L})$ & $\mathrm{LOQ}(\mu \mathrm{g} / \mathrm{L})$ \\
\hline NP & $10-100$ & 0.991 & 6.5 & 0.2 & 1.0 \\
ANP & $10-100$ & 0.993 & 4.6 & 0.3 & 1.0 \\
ANE & $10-100$ & 0.990 & 3.5 & 0.3 & 1.0 \\
FLR & $10-100$ & 0.995 & 4.8 & 0.3 & 1.0 \\
PHE & $10-100$ & 0.992 & 5.4 & 0.7 & 2.0 \\
FLT & $10-100$ & 0.992 & 4.9 & 1.0 & 5.0 \\
PY & $10-100$ & 0.991 & 5.5 & 1.0 & 5.0 \\
CHR & $10-100$ & 0.993 & 5.2 & 1.5 & 5.0 \\
\hline
\end{tabular}

Table S4. Identification of PAHs in tap water, tap water spiked with 10 ng of 8 PAHs, and a soil sample with CDBES-GC-MS. ${ }^{\text {a }}$

\begin{tabular}{ccccc}
\hline PAHs & $\begin{array}{c}\text { Tap water } \\
(\mu \mathrm{g} / \mathrm{L})\end{array}$ & $\begin{array}{c}\text { Spiked tap water } \\
(\mu \mathrm{g} / \mathrm{L})\end{array}$ & $\begin{array}{c}\text { Recoveries }(\%) \\
\text { Soil }(\mu \mathrm{g} / \mathrm{kg})\end{array}$ \\
\hline NP & nq & 0.92 & 92.0 & nd \\
ANY & nq & 0.82 & 82.0 & nd \\
ANE & nq & 0.85 & 85.0 & nq \\
FLR & nq & 0.86 & 86.0 & nd \\
PHE & nq & 0.88 & 88.0 & nd \\
FLT & nq & 0.81 & 81.0 & nd \\
PY & nq & 0.80 & 80.0 & nd \\
CHR & nq & 0.81 & 81.0 & nd \\
a nd, not detected; nq, not quantified. & & \\
\hline
\end{tabular}


Table S5. The comparison of main volatiles (>1.0\%) with different sample preparation techniques.

\begin{tabular}{|c|c|c|c|c|c|c|c|}
\hline \multirow{2}{*}{ No. } & \multirow{2}{*}{$\mathrm{t}_{\mathrm{R}} / \mathrm{min}$} & \multirow{2}{*}{ Name } & \multirow{2}{*}{ Formula } & \multicolumn{4}{|c|}{ Sample preparation techniques/peak area } \\
\hline & & & & CDBES & HS & HS-SPME & DHC \\
\hline 1 & 3.813 & Dimethyl disulfide & $\mathrm{C}_{2} \mathrm{H}_{6} \mathrm{~S}_{2}$ & $2.90 \mathrm{E}+07$ & $1.40 \mathrm{E}+05$ & $1.81 \mathrm{E}+05$ & $1.81 \mathrm{E}+05$ \\
\hline 2 & 6.148 & Butyl isobutyrate & $\begin{array}{c}\mathrm{C}_{8} \mathrm{H}_{16} \mathrm{O} \\
{ }_{2}\end{array}$ & $4.79 \mathrm{E}+07$ & $3.02 \mathrm{E}+04$ & $7.10 \mathrm{E}+05$ & $7.10 \mathrm{E}+05$ \\
\hline 3 & 6.422 & 3-Carene & $\mathrm{C}_{10} \mathrm{H}_{16}$ & $7.95 \mathrm{E}+07$ & $2.98 \mathrm{E}+05$ & $2.01 \mathrm{E}+06$ & $2.01 \mathrm{E}+06$ \\
\hline 4 & 6.619 & Camphene & $\mathrm{C}_{10} \mathrm{H}_{16}$ & $4.44 \mathrm{E}+07$ & $2.50 \mathrm{E}+05$ & $1.53 \mathrm{E}+06$ & $1.53 \mathrm{E}+06$ \\
\hline 5 & 7.124 & (1S)-(1)- $\beta$-Pinene & $\mathrm{C}_{10} \mathrm{H}_{16}$ & $4.65 \mathrm{E}+08$ & $9.50 \mathrm{E}+05$ & $2.24 \mathrm{E}+07$ & $2.24 \mathrm{E}+07$ \\
\hline 6 & 7.357 & $\begin{array}{c}\text { Isopentyl } \\
\text { isobutyrate }\end{array}$ & $\begin{array}{c}\mathrm{C}_{9} \mathrm{H}_{18} \mathrm{O} \\
{ }_{2}\end{array}$ & $3.83 \mathrm{E}+07$ & $7.84 \mathrm{E}+04$ & $3.37 \mathrm{E}+06$ & $3.37 \mathrm{E}+06$ \\
\hline 7 & 7.406 & $\begin{array}{l}\text { 2-Methylbutyl } \\
\text { isobutyrate }\end{array}$ & $\begin{array}{c}\mathrm{C}_{9} \mathrm{H}_{18} \mathrm{O} \\
{ }_{2}\end{array}$ & $1.52 \mathrm{E}+08$ & $5.54 \mathrm{E}+05$ & $1.76 \mathrm{E}+07$ & $1.76 \mathrm{E}+07$ \\
\hline 8 & 7.538 & $\begin{array}{c}\text { 3-Ethyl-2- } \\
\text { pentenoic acid } \\
\text { methyl ester }\end{array}$ & $\begin{array}{c}\mathrm{C}_{8} \mathrm{H}_{14} \mathrm{O} \\
{ }_{2}\end{array}$ & $9.04 \mathrm{E}+07$ & $1.13 \mathrm{E}+05$ & $5.16 \mathrm{E}+06$ & $5.16 \mathrm{E}+06$ \\
\hline 9 & 7.591 & $D$-Limonene & $\mathrm{C}_{10} \mathrm{H}_{16}$ & $1.23 \mathrm{E}+08$ & $5.73 \mathrm{E}+05$ & $1.46 \mathrm{E}+07$ & $1.46 \mathrm{E}+07$ \\
\hline 10 & 8.268 & $\begin{array}{l}\text { 1-Methyl-4-(1- } \\
\text { methylethenyl)- } \\
\text { benzene }\end{array}$ & $\mathrm{C}_{10} \mathrm{H}_{12}$ & $3.39 \mathrm{E}+07$ & $8.42 \mathrm{E}+03$ & $6.21 \mathrm{E}+05$ & $6.21 \mathrm{E}+05$ \\
\hline 11 & 8.358 & $\begin{array}{c}\text { Formic acid linalyl } \\
\text { ester }\end{array}$ & $\begin{array}{c}\mathrm{C}_{11} \mathrm{H}_{18} \mathrm{O} \\
{ }_{2}\end{array}$ & $5.08 \mathrm{E}+07$ & $2.38 \mathrm{E}+04$ & $5.89 \mathrm{E}+05$ & $5.89 \mathrm{E}+05$ \\
\hline 12 & 8.387 & $\begin{array}{l}\text { 2-Ethenyl-1,1- } \\
\text { dimethyl-3- } \\
\text { methylene- } \\
\text { Cyclohexane }\end{array}$ & $\mathrm{C}_{11} \mathrm{H}_{18}$ & $3.03 \mathrm{E}+07$ & $2.77 \mathrm{E}+04$ & $1.84 \mathrm{E}+06$ & $1.84 \mathrm{E}+06$ \\
\hline 13 & 11.191 & $\alpha$-Copaene & $\mathrm{C}_{15} \mathrm{H}_{24}$ & $4.00 \mathrm{E}+07$ & $6.82 \mathrm{E}+04$ & $3.12 E+06$ & $3.12 \mathrm{E}+06$ \\
\hline 14 & 11.618 & Caryophyllene & $\mathrm{C}_{15} \mathrm{H}_{24}$ & $2.09 \mathrm{E}+08$ & $2.92 \mathrm{E}+05$ & $2.09 \mathrm{E}+07$ & $2.09 \mathrm{E}+07$ \\
\hline 15 & 11.925 & Humulene & $\mathrm{C}_{15} \mathrm{H}_{24}$ & $4.83 \mathrm{E}+08$ & $9.14 \mathrm{E}+05$ & $7.19 \mathrm{E}+07$ & $7.19 \mathrm{E}+07$ \\
\hline 16 & 12.061 & $\gamma$-Muurolene & $\mathrm{C}_{15} \mathrm{H}_{24}$ & $6.32 E+07$ & $1.08 \mathrm{E}+05$ & $6.89 \mathrm{E}+06$ & $6.89 E+06$ \\
\hline 17 & 12.192 & $\beta$-Selinene & $\mathrm{C}_{15} \mathrm{H}_{24}$ & $5.78 \mathrm{E}+07$ & $5.46 \mathrm{E}+03$ & $2.40 \mathrm{E}+06$ & $2.40 \mathrm{E}+06$ \\
\hline 18 & 12.253 & $\alpha$-Selinene & $\mathrm{C}_{15} \mathrm{H}_{24}$ & $8.48 \mathrm{E}+07$ & $9.00 \mathrm{E}+04$ & $7.16 \mathrm{E}+06$ & $7.16 \mathrm{E}+06$ \\
\hline 19 & 12.389 & $\gamma$-Muurolene & $\mathrm{C}_{15} \mathrm{H}_{24}$ & $3.92 \mathrm{E}+07$ & $1.54 \mathrm{E}+05$ & $1.04 \mathrm{E}+07$ & $1.04 \mathrm{E}+07$ \\
\hline
\end{tabular}


Table S6. Volatiles in lemonade bubble iced coffee analyzed with CDBES-GC-MS showing the retention time, name, formula, CAS, and percentage of matching score of each compound according to the NIST11 database.

\begin{tabular}{|c|c|c|c|c|c|c|}
\hline No. & $t_{R}(\min )$ & Name & Formula & CAS & Match score (\%) & Area (\%) \\
\hline 1 & 2.244 & Glutaraldehyde & $\mathrm{C}_{5} \mathrm{H}_{8} \mathrm{O}_{2}$ & $111-30-8$ & 65.4 & 0.50 \\
\hline 2 & 2.601 & Isovaleraldehyde & $\mathrm{C}_{5} \mathrm{H}_{10} \mathrm{O}$ & $590-86-3$ & 75.1 & 0.39 \\
\hline 3 & 2.712 & Pentanal & $\mathrm{C}_{5} \mathrm{H}_{10} \mathrm{O}$ & $110-62-3$ & 78.2 & 0.65 \\
\hline 4 & 3.025 & Succindialdehyde & $\mathrm{C}_{4} \mathrm{H}_{6} \mathrm{O}_{2}$ & $638-37-9$ & 67.9 & 0.35 \\
\hline 5 & 3.528 & $\begin{array}{l}\text { 4-Methyl-2- } \\
\text { hexanamine }\end{array}$ & $\mathrm{C}_{7} \mathrm{H}_{17} \mathrm{~N}$ & $105-41-9$ & 76.2 & 1.12 \\
\hline 6 & 4.182 & $\begin{array}{c}\text { 2-Fluoro- } \beta, 3- \\
\text { dihydroxy- } N \text {-methyl- } \\
\text { benzeneethanamine }\end{array}$ & $\mathrm{C}_{9} \mathrm{H}_{12} \mathrm{FNO}_{2}$ & 103439-04-9 & 70.8 & 0.34 \\
\hline 7 & 4.593 & Paromomycin & $\mathrm{C}_{23} \mathrm{H}_{45} \mathrm{~N}_{5} \mathrm{O}_{14}$ & $7542-37-2$ & 76.8 & 1.79 \\
\hline 8 & 5.154 & 2,5-Dimethyl-furan & $\mathrm{C}_{6} \mathrm{H}_{8} \mathrm{O}$ & $625-86-5$ & 69.6 & 0.34 \\
\hline 9 & 7.194 & 2-Thujene & $\mathrm{C}_{10} \mathrm{H}_{16}$ & 28634-89-1 & 79.2 & 1.06 \\
\hline 10 & 7.327 & $(1 R)-(+)-\alpha-$-Pinene & $\mathrm{C}_{10} \mathrm{H}_{16}$ & 7785-70-8 & 91.8 & 5.31 \\
\hline 11 & 7.565 & $\begin{array}{l}\text { 5-Methyl-2- } \\
\text { furancarboxaldehyde }\end{array}$ & $\mathrm{C}_{6} \mathrm{H}_{6} \mathrm{O}_{2}$ & $620-02-0$ & 78.9 & 0.34 \\
\hline 12 & 7.928 & $\begin{array}{l}\text { 2,6,6-trimethoxy-2- } \\
\text { vinyltetrahydropyran }\end{array}$ & $\mathrm{C}_{10} \mathrm{H}_{18} \mathrm{O}$ & $7392-19-0$ & 73.6 & 0.89 \\
\hline 13 & 8.056 & $\beta$-Pinene & $\mathrm{C}_{10} \mathrm{H}_{16}$ & $127-91-3$ & 88.2 & 6.02 \\
\hline 14 & 8.25 & $\beta$-Myrcene & $\mathrm{C}_{10} \mathrm{H}_{16}$ & $123-35-3$ & 83.3 & 1.72 \\
\hline 15 & 8.519 & 3-Carene & $\mathrm{C}_{10} \mathrm{H}_{16}$ & $13466-78-9$ & 69.5 & 0.55 \\
\hline 16 & 8.674 & 1,4-Cineole & $\mathrm{C}_{10} \mathrm{H}_{18} \mathrm{O}$ & $470-67-7$ & 90.4 & 2.20 \\
\hline 17 & 8.727 & $\alpha$-Terpinene & $\mathrm{C}_{10} \mathrm{H}_{16}$ & $99-86-5$ & 85.3 & 0.87 \\
\hline 18 & 8.793 & o-Cymene & $\mathrm{C}_{10} \mathrm{H}_{14}$ & $527-84-4$ & 85.7 & 1.08 \\
\hline 19 & 9.014 & $D$-Limonene & $\mathrm{C}_{10} \mathrm{H}_{16}$ & $5989-27-5$ & 92.1 & 32.69 \\
\hline 20 & 9.177 & Isopulegol & $\mathrm{C}_{10} \mathrm{H}_{18} \mathrm{O}$ & 89-79-2 & 77.5 & 1.02 \\
\hline 21 & 9.425 & $\gamma$-Terpinene & $\mathrm{C}_{10} \mathrm{H}_{16}$ & $99-85-4$ & 93.3 & 7.32 \\
\hline 22 & 9.584 & $\begin{array}{l}(E) \text {-10-Heptadecen-8- } \\
\text { ynoic acid methyl ester }\end{array}$ & $\mathrm{C}_{18} \mathrm{H}_{30} \mathrm{O}_{2}$ & $16714-85-5$ & 69.4 & 0.89 \\
\hline 23 & 9.827 & $\begin{array}{l}\text { 1-Methyl-4-(1- } \\
\text { methylethenyl)- } \\
\text { benzene }\end{array}$ & $\mathrm{C}_{10} \mathrm{H}_{12}$ & $1195-32-0$ & 84.8 & 0.70 \\
\hline 24 & 9.897 & Terpinolene & $\mathrm{C}_{10} \mathrm{H}_{16}$ & $586-62-9$ & 89.7 & 0.96 \\
\hline 25 & 9.955 & $\begin{array}{c}\text { 1,2-15,16- } \\
\text { Diepoxyhexadecane }\end{array}$ & $\mathrm{C}_{16} \mathrm{H}_{30} \mathrm{O}_{2}$ & I & 75.6 & 0.41 \\
\hline
\end{tabular}




\begin{tabular}{|c|c|c|c|c|c|c|}
\hline 26 & 10.021 & Linalool & $\mathrm{C}_{10} \mathrm{H}_{18} \mathrm{O}$ & $78-70-6$ & 89.0 & 1.27 \\
\hline 27 & 10.299 & Fencho & $\mathrm{C}_{10} \mathrm{H}_{18} \mathrm{O}$ & $1632-73-1$ & 92.1 & 2.41 \\
\hline 28 & 10.604 & $\begin{array}{l}\text { 1-Methyl-4-(1- } \\
\text { methylethyl)-3- } \\
\text { cyclohexen-1-ol }\end{array}$ & $\mathrm{C}_{10} \mathrm{H}_{18} \mathrm{O}$ & $586-82-3$ & 84.2 & 0.78 \\
\hline 29 & 10.838 & Paromomycin & $\mathrm{C}_{23} \mathrm{H}_{45} \mathrm{~N}_{5} \mathrm{O}_{14}$ & $7542-37-2$ & 67.6 & 0.57 \\
\hline 30 & 10.962 & Isoborneol & $\mathrm{C}_{10} \mathrm{H}_{18} \mathrm{O}$ & $10385-78-1$ & 79.2 & 0.45 \\
\hline 31 & 11.112 & Borneol & $\mathrm{C}_{10} \mathrm{H}_{18} \mathrm{O}$ & $507-70-0$ & 86.6 & 1.39 \\
\hline 32 & 11.284 & (-)-Terpien-4-ol & $\mathrm{C}_{10} \mathrm{H}_{18} \mathrm{O}$ & 20126-76-5 & 91.8 & 2.06 \\
\hline 33 & 11.487 & $\alpha$-Terpineol & $\mathrm{C}_{10} \mathrm{H}_{18} \mathrm{O}$ & $98-55-5$ & 94.0 & 5.63 \\
\hline 34 & 11.81 & $\begin{array}{c}5- \\
\text { Hydroxymethylfurfural }\end{array}$ & $\mathrm{C}_{6} \mathrm{H}_{6} \mathrm{O}_{3}$ & $67-47-0$ & 89.6 & 3.47 \\
\hline 35 & 11.977 & Nerol & $\mathrm{C}_{10} \mathrm{H}_{18} \mathrm{O}$ & $106-25-2$ & 75.9 & 3.00 \\
\hline 36 & 12.326 & Geraniol & $\mathrm{C}_{10} \mathrm{H}_{18} \mathrm{O}$ & $106-24-1$ & 76.8 & 0.66 \\
\hline 37 & 12.441 & Citral & $\mathrm{C}_{10} \mathrm{H}_{16} \mathrm{O}$ & $5392-40-5$ & 85.3 & 0.82 \\
\hline 38 & 12.613 & $\begin{array}{l}\text { 3,7,11-Trimethyl-1- } \\
\text { dodecanol }\end{array}$ & $\mathrm{C}_{15} \mathrm{H}_{32} \mathrm{O}$ & $6750-34-1$ & 71.8 & 0.38 \\
\hline 39 & 13.033 & $\begin{array}{c}\text { 2-[(Z)-9- } \\
\text { Octadecenyloxy] } \\
\text { ethanol }\end{array}$ & $\mathrm{C}_{20} \mathrm{H}_{40} \mathrm{O}_{2}$ & $5353-25-3$ & 75.3 & 0.33 \\
\hline 40 & 13.236 & $\begin{array}{l}\text { cis-10-Heptadecenoic } \\
\text { acid }\end{array}$ & $\mathrm{C}_{17} \mathrm{H}_{32} \mathrm{O}_{2}$ & $29743-97-3$ & 69.4 & 0.40 \\
\hline 41 & 13.77 & Neryl acetate & $\mathrm{C}_{12} \mathrm{H}_{20} \mathrm{O}_{2}$ & $141-12-8$ & 92.0 & 1.16 \\
\hline 42 & 14.009 & Geranyl acetate & $\mathrm{C}_{12} \mathrm{H}_{20} \mathrm{O}_{2}$ & $105-87-3$ & 90.6 & 0.70 \\
\hline 43 & 14.861 & Caryophyllene & $\mathrm{C}_{15} \mathrm{H}_{24}$ & $87-44-5$ & 85.7 & 0.53 \\
\hline 44 & 14.998 & $\alpha$-Bergamotene & $\mathrm{C}_{15} \mathrm{H}_{24}$ & 17699-05-7 & 87.8 & 0.42 \\
\hline 45 & 15.873 & $\beta$-Bisabolene & $\mathrm{C}_{15} \mathrm{H}_{24}$ & 495-61-4 & 83.6 & 0.42 \\
\hline 46 & 19.988 & Caffeine & $\mathrm{C}_{8} \mathrm{H}_{10} \mathrm{~N}_{4} \mathrm{O}_{2}$ & 58-08-2 & 77.4 & 0.46 \\
\hline
\end{tabular}

Table S7. Volatiles found in the urine analyzed with CDBES-GC-MS showing the retention time, name, formula, CAS, and percentage of matching score of each compound according to the NIST11 database.

\begin{tabular}{|c|c|c|c|c|c|c|}
\hline No. & $t_{R}(\min )$ & Name & Formula & CAS & Match score (\%) & Area (\%) \\
\hline & & $N-[(4-$ & & & & \\
\hline 1 & 5.889 & $\begin{array}{l}\text { Hydroxy)hydrocinnamoyl]- } \\
\text { benzeneethanamine }\end{array}$ & $\mathrm{C}_{17} \mathrm{H}_{19} \mathrm{NO}_{2}$ & 106827-59-2 & 64.9 & 14.4 \\
\hline 2 & 6.644 & $\begin{array}{l}\text { 2-Methylbicyclo[4.3.0]non- } \\
\text { 1(6)-ene }\end{array}$ & $\mathrm{C}_{10} \mathrm{H}_{16}$ & 60223-07-6 & 74.8 & 2.42 \\
\hline 3 & 6.767 & $\begin{array}{c}2- \\
\text { Aminophenanthroimidazole }\end{array}$ & $\mathrm{C}_{15} \mathrm{H}_{11} \mathrm{~N}_{3}$ & 37052-13-4 & 56.1 & 5.60 \\
\hline
\end{tabular}




\begin{tabular}{|c|c|c|c|c|c|c|}
\hline 4 & 7.41 & Benzene, 1,4-dichloro- & $\mathrm{C}_{6} \mathrm{H}_{4} \mathrm{Cl}_{2}$ & $106-46-7$ & 87.1 & 7.25 \\
\hline 5 & 7.706 & $\begin{array}{c}\text { 2,5-Difluoro- } \beta, 3,4- \\
\text { trihydroxy- } N \text {-methyl- } \\
\text { benzeneethanamine }\end{array}$ & $\mathrm{C}_{9} \mathrm{H}_{11} \mathrm{~F}_{2} \mathrm{NO}_{3}$ & $152434-78-1$ & 58.0 & 1.63 \\
\hline 6 & 7.882 & Pterin-6-carboxylic acid & $\mathrm{C}_{7} \mathrm{H}_{5} \mathrm{~N}_{5} \mathrm{O}_{3}$ & $948-60-7$ & 57.4 & 1.00 \\
\hline 7 & 8.095 & $\gamma$-Chlorobutyrophenone & $\mathrm{C}_{10} \mathrm{H}_{11} \mathrm{ClO}$ & $939-52-6$ & 56.1 & 2.26 \\
\hline 8 & 8.403 & Nonanal & $\mathrm{C}_{9} \mathrm{H}_{18} \mathrm{O}$ & $124-19-6$ & 74.9 & 6.77 \\
\hline 9 & 8.933 & $\begin{array}{c}\text { 1,5,5-Trimethyl-6- } \\
\text { methylene-cyclohexene }\end{array}$ & $\mathrm{C}_{10} \mathrm{H}_{16}$ & $514-95-4$ & 77.7 & 1.04 \\
\hline 10 & 8.936 & D-(+)-Camphor & $\mathrm{C}_{10} \mathrm{H}_{16} \mathrm{O}$ & $464-49-3$ & 78.1 & 2.41 \\
\hline 11 & 9.059 & Neoisothujyl alcohol & $\mathrm{C}_{10} \mathrm{H}_{18} \mathrm{O}$ & $31187-53-8$ & 69.3 & 1.11 \\
\hline 12 & 9.157 & Isobornyl formate & $\mathrm{C}_{11} \mathrm{H}_{18} \mathrm{O}_{2}$ & $1200-67-5$ & 76.0 & 1.64 \\
\hline 13 & 9.379 & Dodecane & C12H26 & $112-40-3$ & 79.5 & 3.05 \\
\hline 14 & 10.092 & Actinobolin & $\mathrm{C}_{13} \mathrm{H}_{20} \mathrm{~N}_{2} \mathrm{O}_{6}$ & $24397-89-5$ & 57.4 & 1.28 \\
\hline 15 & 10.346 & Tridecane & $\mathrm{C}_{13} \mathrm{H}_{28}$ & $629-50-5$ & 81.9 & 2.60 \\
\hline 16 & 10.949 & $\begin{array}{l}\text { 1,6-Dichloro-1,5- } \\
\text { cyclooctadiene }\end{array}$ & $\mathrm{C}_{8} \mathrm{H}_{10} \mathrm{Cl}_{2}$ & $29480-42-0$ & 73.2 & 1.31 \\
\hline 17 & 11.195 & $\alpha$-ylangene & $\mathrm{C}_{15} \mathrm{H}_{24}$ & / & 76.8 & 2.12 \\
\hline 18 & 11.252 & Tetradecane & $\mathrm{C}_{14} \mathrm{H}_{30}$ & $629-59-4$ & 79.9 & 3.75 \\
\hline 19 & 11.519 & Longifolene & $\mathrm{C}_{15} \mathrm{H}_{24}$ & $475-20-7$ & 90.4 & 7.05 \\
\hline 20 & 11.568 & 4-Epi-cubedol & $\mathrm{C}_{15} \mathrm{H}_{26} \mathrm{O}$ & / & 68.4 & 1.43 \\
\hline 21 & 11.683 & 8,9-Epoxyacorenon-B & $\mathrm{C}_{15} \mathrm{H}_{24} \mathrm{O}_{2}$ & $61050-91-7$ & 67.0 & 2.50 \\
\hline 22 & 11.794 & $\begin{array}{l}\text { 3,7,11-Trimethyl-1- } \\
\text { dodecanol }\end{array}$ & $\mathrm{C}_{15} \mathrm{H}_{32} \mathrm{O}$ & $6750-34-1$ & 66.2 & 1.40 \\
\hline 23 & 11.954 & $\begin{array}{l}\text { 2,5-Cyclohexadiene-1,4- } \\
\text { dione }\end{array}$ & $\mathrm{C}_{14} \mathrm{H}_{20} \mathrm{O}_{2}$ & $719-22-2$ & 90.2 & 8.00 \\
\hline 24 & 12.052 & Cubedol & $\mathrm{C}_{15} \mathrm{H}_{26} \mathrm{O}$ & I & 64.2 & 1.69 \\
\hline 25 & 12.105 & tert-Hexadecanethiol & $\mathrm{C}_{16} \mathrm{H}_{34} \mathrm{~S}$ & 25360-09-2 & 66.5 & 1.86 \\
\hline 26 & 12.155 & 8-Cedren-13-ol & $\mathrm{C}_{15} \mathrm{H}_{24} \mathrm{O}$ & 18319-35-2 & 66.6 & 1.48 \\
\hline 27 & 12.245 & 4-Epi-cubedol & $\mathrm{C}_{15} \mathrm{H}_{26} \mathrm{O}$ & / & 67.9 & 1.32 \\
\hline 28 & 12.306 & Butylated hydroxytoluene & $\mathrm{C}_{15} \mathrm{H}_{24} \mathrm{O}$ & $128-37-0$ & 78.0 & 1.85 \\
\hline 29 & 12.433 & Fenretinide & $\mathrm{C}_{26} \mathrm{H}_{33} \mathrm{NO}_{2}$ & 65646-68-6 & 60.4 & 2.09 \\
\hline 30 & 12.507 & $\begin{array}{c}\text { Octahydro-4,8,8,9- } \\
\text { tetramethyl-, (+)- 1,4- } \\
\text { methanoazulen-7(1H)-one }\end{array}$ & $\mathrm{C}_{15} \mathrm{H}_{24} \mathrm{O}$ & 18319-28-3 & 62.6 & 1.33 \\
\hline 31 & 12.581 & 1-Heptatriacotanol & $\mathrm{C}_{37} \mathrm{H}_{76} \mathrm{O}$ & $105794-58-9$ & 65.3 & 3.19 \\
\hline 32 & 12.913 & $\begin{array}{l}\text { 3,7,11-Trimethyl-1- } \\
\text { dodecanol }\end{array}$ & $\mathrm{C}_{15} \mathrm{H}_{32} \mathrm{O}$ & $6750-34-1$ & 62.0 & 1.85 \\
\hline 33 & 13.274 & $\begin{array}{l}\text { 11,14-Eicosadienoic acid } \\
\text { methyl ester }\end{array}$ & $\mathrm{C}_{21} \mathrm{H}_{38} \mathrm{O}_{2}$ & $2463-02-7$ & 55.6 & 1.30 \\
\hline
\end{tabular}




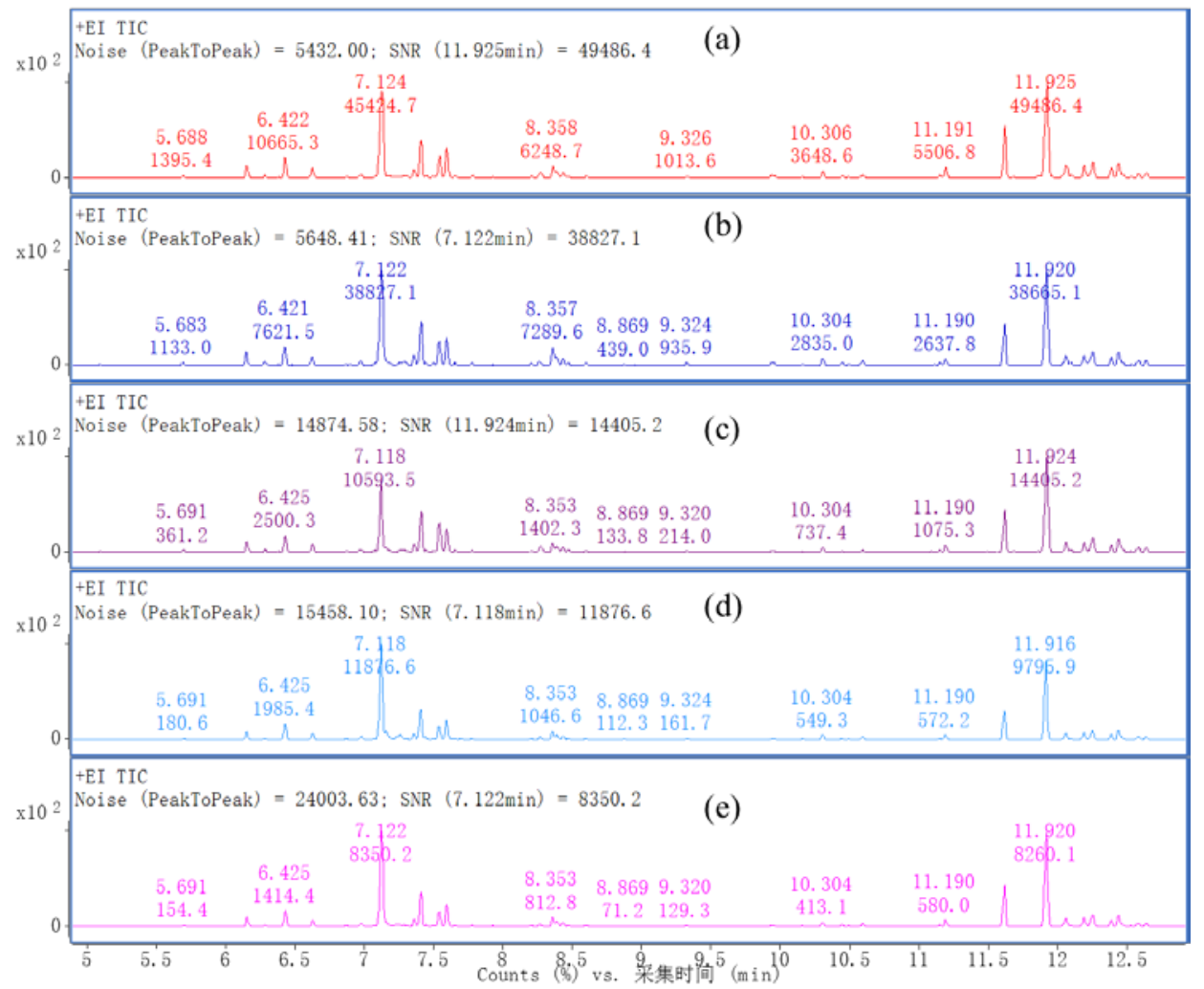

Figure S1. The overall SNR trend of bubbling extraction with different carrier gases:

(a) $\mathrm{CO}_{2}$, (b) $\mathrm{CH}_{4}$, (c) $\mathrm{N}_{2}$, (d) $\mathrm{He}$, and (e) Ar. 

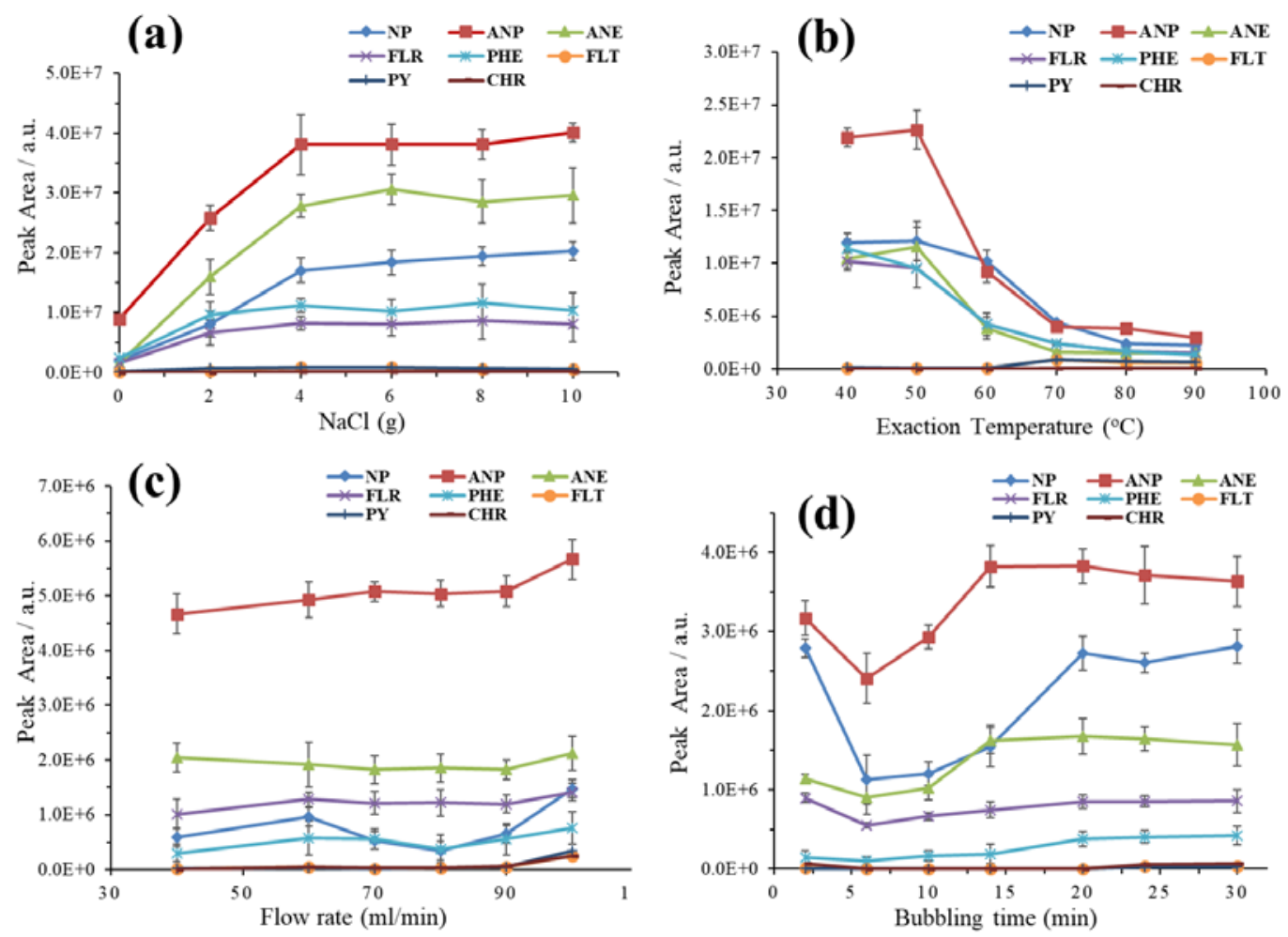

Figure S2. Influence of the parameters on the $\mathrm{CO}_{2}$ bubbling extraction efficiency using $50 \mu \mathrm{g} / \mathrm{mL}$ standard PAHs in a spiked water solution. (a) ionic strength effects: extraction temperature of $50{ }^{\circ} \mathrm{C}$, bubbling time of $15 \mathrm{~min}$ and $\mathrm{CO}_{2}$ flow rate of $100 \mathrm{~mL} / \mathrm{min}(n=$ 3); (b) extraction temperature effects: $4.0 \mathrm{~g}$ of $\mathrm{NaCl}$, bubbling time of $15 \mathrm{~min}$ and $\mathrm{CO}_{2}$ flow rate of $100 \mathrm{~mL} / \mathrm{min}(n=3)$; (c) effects of $\mathrm{CO}_{2}$ flow rate: extraction temperature of $50{ }^{\circ} \mathrm{C}, 4.0 \mathrm{~g}$ of $\mathrm{NaCl}$ and bubbling time of $15 \mathrm{~min}(n=3)$; and (d) effects of bubbling time: $\mathrm{CO}_{2}$ flow rate of $100 \mathrm{~mL} / \mathrm{min}$, extraction temperature of $50{ }^{\circ} \mathrm{C}$ and $4.0 \mathrm{~g}$ of $\mathrm{NaCl}$ $(n=3)$.

\section{References}

(1) O'Dowd, C. D.; Facchini, M. C.; Cavalli, F.; Ceburnis, D.; Mircea, M.; Decesari, S.; Fuzzi, S.; Yoon, Y. J.; Putaud, J. P. Biogenically driven organic contribution to 
marine aerosol. Nature 2004, 431, 676-680.

(2) Rettberg, N.; Biendl, M.; Garbe, L.-A. Hop aroma and hoppy beer flavor: chemical backgrounds and analytical tools-a review. J. Am. Soc. Brew. Chem. 2018, 76, 1-20.

(3) Aberl, A.; Coelhan, M. Determination of Volatile Compounds in Different Hop Varieties by Headspace-Trap GC/MS-In Comparison with Conventional Hop Essential Oil Analysis. J. Agr. Food Chem. 2012, 60, 2785-2792.

(4) Kuligowski, J.; Escobar, J.; Quintás, G.; Lliso, I.; Torres-Cuevas, I.; Nuñez, A.; Cubells, E.; Rook, D.; van Goudoever, J. B.; Vento, M. Analysis of lipid peroxidation biomarkers in extremely low gestational age neonate urines by UPLC-MS/MS. Anal. Bioanal. Chem. 2014, 406, 4345-4356. 